\begin{tabular}{|c|c|}
\hline \multirow{3}{*}{ 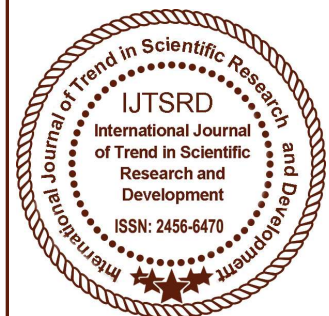 } & $\begin{array}{l}\text { International Journal of Trend in Scientific } \\
\text { Research and Development (IJTSRD) }\end{array}$ \\
\hline & International Open Access Journal \\
\hline & ISSN No: 2456 - 6470 | www.ijtsrd.com | Volume - 1 | Issue - 5 \\
\hline
\end{tabular}

\title{
FtsZ, A Tubulin Homologue Spatiotemporally Regulates the Bacterial Cell Division
}

\author{
Aijaz Rashid* \\ Postdoctoral Research Fellow, \\ National Institutes of Health, NIH, USA \\ *Corresponding author: clbaijaz@gmail.com
}

\author{
Shazia Ahad \\ Indian Institute of Technology(IIT) Bombay, \\ Maharastra, India
}

\begin{abstract}
Most common pattern of Bacterial division is binary fission. Bacterial division occurs by the construction of Z-ring at appropriate time and at a defined site. FtsZ is the major component of Z-ring formation, and the polymerization of Fts $Z$ is regulated in space and time by negative and positive regulators of FtsZ assembly. In the bacterial cell positive and negative regulators help in attaining a critical concentration gradient, such that at mid cell the critical concentration required for Z-ring formation is lowest, thus Z-ring formation takes place at this site only.
\end{abstract}

\section{INTRODUCTION:}

Bacterial growth and division occurs by many ways, some bacteria grow double of their size and by multiple fission produce multiple offspring, others reproduce by means of budding and in some cases internal offspring are produced with in large mother cell. Among all these cell divisions, binary Fission is the most common method of division in prokaryotes. During binary Fission cell grows double of its size and then splits in to two physiologically and genetically identical cells. Why we need to understand bacterial cell division? This is important because understanding of bacterial cell division gives us a chance to find out the way to alter the pathways, which will help to design new chemicals or novel antibiotics that specifically target the mechanism of cell division in bacteria.

Although binary fission appears simple, it is complex and highly regulated process. Septation takes place after the competition of DNA replication and nucleoid segregations. Main protein involved in bacterial cell division is FtsZ, a tubulin homologue. With the help of many accessory proteins, FtsZ form a Z-Ring that in turn forms a divisome complex. FtsZ is $40 \mathrm{kDa}$ protein having intrinsic GTPase activity. Z-ring is positioned in such a way at the medial position of the cell, that there is no damage/disturbance to separating DNA.

Binary fission takes place by means of contractile ring (Z-ring) which encircles the medial portion of the cell. The main protein of Z-Ring is FtsZ and is conserved in most bacteria and archea. Z-Ring accessory proteins anchor it to the cell membrane, so that force generated during Z-ring contraction is transferred to the cell membrane. Thus, resulting in the construction of cell membrane and cell wall ultimately leading to division of cytoplasm in to two daughter cells.

\section{Discovery and abundance of FtsZ in prokaryotes:}

FtsZ is a tubulin homolog, which was discovered in temperature sensitive bacteria. At higher temperature these bacteria attain filamentous shape and were incapable of growth. FtsZ is almost universally conserved protein throughout the bacterial kingdom. It is also present in eukaryotic organelles like mitochondria and plastids of several groups of eukaryotes, an indication of symbiotic relationship between prokaryotic and eukaryotic cells (1).

In Caulobacter crescentus there is variation in the synthesis and degradation (proteolysis) of FtsZ, 
depending on the cell cycle stage and type of cell (2). In case of two well studied bacteria Escherichia Coli and Bacillus subtilis no significant change in the FtsZ level has been found throughout cell division or under different growth conditions. So there must be strict regulation in FtsZ assembly (3). Positive regulators decrease the critical concentration of FtsZ for Z-Ring formation while negative regulators increase the critical concentration for Z-Ring formation. Negative regulators determine the appropriate time and position of Z-ring formation.

\section{Understanding of Z-ring assembly and dynamics through in vitro and in vivo experiments:}

In vivo as well as in vitro experiments played a major role to understand the mechanism of Z-ring assembly and disassembly. The nature of FtsZ monomer, various proteins and factors responsible for association and disassembly of FtsZ in in vitro, helped us in associating these properties to biological mechanism of cell division. FtsZ has GTPase activity and has ability to assemble into protofilaments, bundles and mini-rings. During in vitro experiments it was found that there is direct correlation of FtsZ concentration and GTPase activity. From this one can predict one subunit may help in cleaving GTP of another subunit by associating with it. It was found by structural analysis that FtsZ like tublin contain nucleotide binding motif. It was found that in FtsZ there is interaction of T7-loope region with the guanine nucleotide on adjacent subunit, resulting in the formation of active site of GTP hydrolysis. Thus, it is a good example of correlating the in vitro observation to in vivo fact or phenomenon. More than $75 \%$ of FtsZ in cells is present as multimers with more than 20 subunits. These multimers continuously exchange subunits as was found by FRET analysis, thus suggesting high dynamicity of Z-ring. Protofilaments in test tube can form polymers in presence of $\mathrm{Mg}^{+}, \mathrm{Ca}^{+}$, and DEAE-dextran suggesting that in biological system also, many ions by means of electrostatatic interaction might help in FtsZ ring formation. The Protofilaments of FtsZ on dissociation of GTP to GDP or GDP+Pi attain a bent conformation, thus we can correlate that in vivo this bending may be responsible for mechanical force generation to help in Z-ring constriction. Most of bacteria possess almost constant concentration of FtsZ, so it is role of positive and negative regulators to favor assembly of FtsZ in to Z-ring at appropriate time and at proper position in cell. In the cell positive and negative regulators help in attaining a critical concentration gradient, such that at mid cell the critical concentration required for Z-ring formation is lowest. By increasing the FtsZ concentration multiple rings at mid cell and poles were formed. Thus we can say that FtsZ concentration is limiting factor for Zring formation. As positive regulators decrease the critical concentration of Z-ring formation at mid cell so FtsZ monomers start assembling, and by cooperative assembly of other monomers lead to formation of contractible Z-ring, which ultimately lead to cell division (fig 1).

\section{Critical concentration gradient model of $\mathrm{Z}$-ring formation:}

During in vitro studies it has been found that FtsZ starts assembling at $0.5-1 \mu \mathrm{M}$ concentration, while Intracellular concentration of FtsZ is 3-10 $\mu \mathrm{M}$ (3). The comparing of in vitro and in vivo data of FtsZ assembly indicates that in vivo assembly of FtsZ assembly may be prevented by negative regulators. The critical concentration gradient model is helpful in understanding the mechanism of Z-ring formation. According to this model during the appropriate division stage of bacteria, a critical concentration gradient is formed for FtsZ assembly. The distribution of positive and negative regulators of Z-ring formation in cell is not random but well programmed. The positive and negative regulators in cell lead to instantaneous critical concentration gradient from mid cell to the poles. The critical concentration of FtsZ required for Z-ring assembly at the centre of cell is less than that at the poles. So FtsZ starts polymerizing at cell centre and afterwards by cooperative association of more and more monomers leads to the Z-ring formation. So, initiation of polymerization acts as seed for futhur FtsZ polymerization. Simultaneously, during Z-ring formation the recruitment of other accessory proteins takes place leading to the formation of a stable and functional divisome complex. 


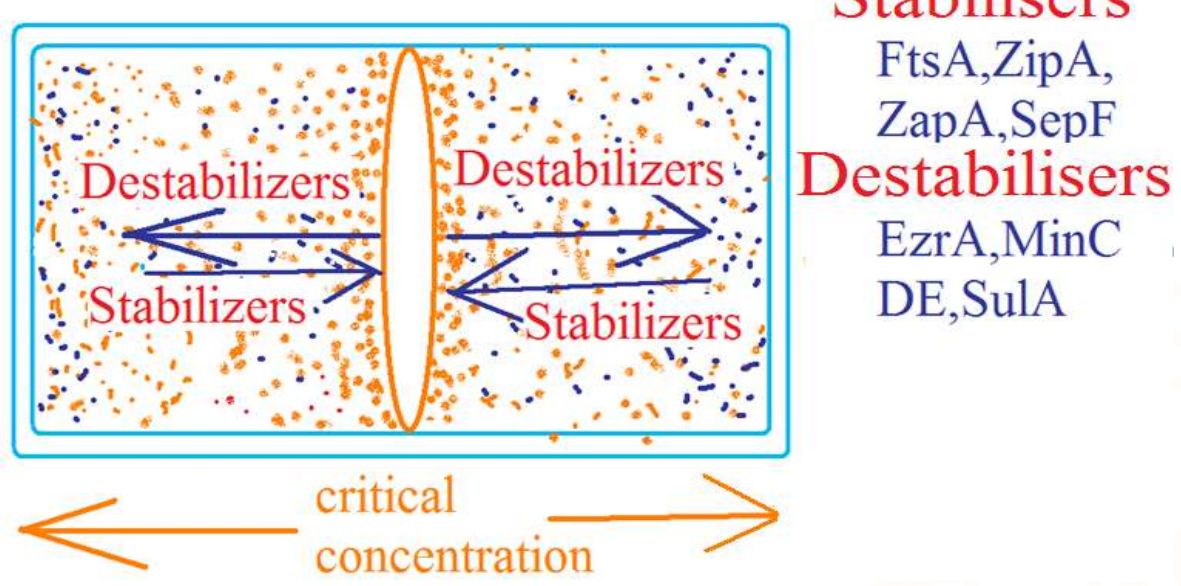

Figure 1: Schematic representation of the Z-ring assembly by critical concentration gradient formation: Critical concentration gradient model is helpful in understanding the mechanism of Z-ring formation. The distribution of positive and negative regulators of Z-ring formation in cell is not random but well programmed. The positive and negative regulators in cell lead to a critical concentration gradient from mid cell to the poles. The critical concentration of FtsZ required for Z-ring assembly at the centre of cell is less than that at the poles. So FtsZ starts polymerizing at cell centre and afterwards by cooperative association of more and more monomers it leads to the Z-ring formation. Simultaneously, during Z-ring formation the recruitment of other accessory proteins takes place, leading to the formation of a stable divisome complex. Positive regulators shown as yellow dots and negative regulators as blue dots.

Regulators of FtsZ ring formation: There are two categories of proteins involved in bacterial cell division, one is positive regulator of Fts Z assembly and other is negative regulator. Bacterial division involves the balance of these regulators. The antagonistic role of these two categories of proteins helps in stability and dynamicity of Z-Ring. The Positive regulators help in Z-Ring formation while negative regulators try to dissociate it, thus establishing equilibrium. The dissociation caused by negative regulators is mechanistic cause of Z-Ring dynamicity.

Positive regulators: The positive regulators decrease the critical concentration of Z-Ring formation, thus they enhance the capacity of FtsZ to form a stable divisome complex. Z-ring formed by the FtsZ is not stable, so recruitment of other downstream proteins takes place by FtsZ resulting in stable and contractible Z-ring formation. Various positive regulators of Zring formation are FtsA, SepF, ZipA and ZapA.

FtsA: It is a widely conserved $47 \mathrm{kDa}$ protein. It is the first protein to be recruited to Z-ring. It is member of Actin/Hexokinase/HSP70 super family (4). FtsA is ATP binding protein. The FtsA purified from Streptococcus pneumonia can bind various nucleotide phosphates according to length of phosphate moiety that means ATP and GTP are preferred over ADP and GDP which in turn are preferred over AMP and GMP (5). FtsA also plays a role in sporulation in Bacillus subtilis ( 6 ) and by means of various two hybrid assays it has been shown that FtsA can interact with itself. The FtsA has a conserved $\mathrm{C}$ - terminal membrane targeting sequence (MTS),deletion or mutation in MTS abolishes the FtsA capacity to interact with cell membrane as well as its self assembly (7). There is specific ratio of FtsZ and FtsA in Escherichia coli $1: 100(8)$ while in the Bacillus subtilis it is $1: 5(6)$, this ratio is important for bacterial cell, any imbalance of this ratio would inhibit bacterial cell division.

ZipA: ZipA is distributed throughout the inner cell membrane and gets concentrated at division site by direct interacting with FtsZ $(9 ; 10)$. The ZipA is recruited to the Z-Ring by direct interaction with FtsZ and is independent of the FtsA $(9 ; 10)$. The ZipA can form heterodimers with FtsZ (11). ZipA also form homodimer as found by two-hybrid assay (12). ZipA has rare membrane topology as its transmembrane anchor is at the amino terminus and C-terminal domain is in the cytosol (3). Same membrane 
topology is shared by division protein extra Z-rings A (EzrA) in Bacillus subtilis (13). ZipA is formed of five domains, amino acid 1-6 are charged periplasmic domains, amino acid 7-28 are transmembrane segments, amino acid 86-185 are called the $\mathrm{P} \backslash \mathrm{Q}$ domains and amino acid 186-328 are globular domains. The globular domains of ZipA binds the Cterminal tail of FtsZ $(9-11 ; 14)$. ZipA was identified by affinity blotting as FtsZ interacting proteins (9). ZipA Localizes to the Z-ring in an FtsZ dependent, but its localization is FtsA-independent manner. The over expression of ZipA abolishes cell division $(9 ; 10)$. The structural studies have revealed that $\mathrm{C}$ terminal domain of ZipA consists of a six-stranded anti-parallel $\beta$-sheets laid against three $\alpha$-helices, with a core domain containing a $\beta-\alpha-\beta$ fold. This domain forms the hydrophobic cleft, to which binds $\mathrm{C}$ terminal 20 amino acids of FtsZ as found by cocrystal structure (3). ZipA contains sequences elements that resemble microtubule binding motifs in Tau, Map2 and Map4 (15). During in vitro experimentation the C-terminal of ZipA promotes the assembly and bundling of FtsZ into protofilaments and sheets by lateral alignment $(14 ; 15)$.

ZapA: ZapA is $10 \mathrm{Kd}$ cytoplasmic protein of 85 amino acid residue. ZapA Protein was identified as a protein whose overexpression can overcome lethality induced by MinD over expression(negative regulator) in Bacillus Subtitles. Deletion mutants of ZapA does not show any specific phenotype abnormality both in Escherichia coli and Bacillus Subtilis (10). These mutants show conditional lethality in various back grounds i,e in presence of mutation /deletion of other positive regulators or in cells with artificially lowered FtsZ Levels (16).

SepF: SepF helps in correct septum formation in Bacillus subtilis (17). The SepF is encoded by $Y l m F$ gene, in vivo sepF interacts with itself as well as the FtsZ and its localization to the Z-Ring depends on the FtsZ $(3 ; 17)$. The SepF is synthetically lethal with FtsA, suggesting that it acts early during the assembly of Z-Ring (3;17). Over production of SepF in FtsA null mutants restore normal Z-ring formation. Thus, SepF overexpression complements FtsA protein absence.

\section{Negative regulators of $\mathrm{Z}$-Ring assembly:}

Negative regulators increase the critical concentration of FtsZ for Z-Ring formation. Negative regulators thus prevent septum formation at inappropriate location and at odd cell division stage/timing.

MinCDE: These proteins were identified by Escherichia Coli mutants that caused aberrant septation at cell poles, leads to mini-cell formation (18). Min C and MinD Proteins together repress the FtsZ ring assembly and hence cell division at polar sites (18). MinB operon codes for three genes MinC, MinD and MinE. The cellular distribution of MinC was determined by fusing it to green fluorescent protein. These studies confirmed that GFP-MinC is concentrated at the polar cap of a single pole in each cell. GFP-MinC does not remains at specific pole but oscillates between poles depending on MinD and MinE (19). When MinC is Present at a given cell pole, it destabilizes the FtsZ polymerization prior to the formation of the Z-Ring structure thus inhibiting polar division (19). MinD activates the inhibitory action of MinC (20). The presence of 8-12 amino acid membrane targeting sequence at the $\mathrm{C}$-terminal is responsible for tethering the MinCD to the membrane.

SulA: SulA is a soluble SOS response protein. SulA binds directly with FtsZ and inhibits its assembly $(21 ; 22)$. The SulA is a cell division inhibitor that is produced in response to DNA damage and leads to inhibition of Z-rings formation, it even causes the disassembly of existing Z-rings at the time of DNA damage (23). In vitro SulA increases the critical concentration of assembly five times (24). SulA binds to C-terminal of FtsZ and sequester them thus increasing the critical concentration of assembly $(25 ; 26)$.

EzrA: EzrA was discovered as extra-genic suppressor of a temperature sensitive FtsZ-GFP (27). EzrA was identified as negative regulator of Z-ring assembly. EzrA is present in lower GC-content gram positive bacteria (28). EzrA is $62 \mathrm{kd}$ protein found in bacillus subtilis (27). EzrA is distributed all over the plasma membrane. The inhibition of cell division caused by overexpression of MinCD is suppressed by EzrA null mutation (27). EzrA -GFP is found distributed throughout the cell membrane and is also concentrated in Z-ring in FtsZ dependent manner. EzrA mutation leads to extra Z-ring formation at the cell poles, suggesting its role similar to Min proteins 
(29). So, we can say EzrA prevents aberrant Z-ring formation at cell poles thus economizing the use of positive regulator proteins for medial cell division to take place. EzrA lacking cells are longer than wild type cells and take longer time for division $(27 ; 30 ; 31)$.Thus EzrA is acting as resource allocator of cell. Another group found that EzrA mutated cells growing in conditions where polar division does not take place, still take longer time to divide $(27 ; 30)$. So they propose that increased cell division time is due to enhanced stability achieved to medial Z-ring in the absence of EzrA. Future support in this direction is that in absence of EzrA the turnover of FtsZ in the Zring is reduced (32). EzrA decreases the FtsZ polymerization and destabilizes the preformed FtsZ protofilaments (33). There is electrostatic interaction between FtsZ and EzrA.

Nucleoid occlusion: According to nucleoid occlusion model nucleoid and its associated transcription, translation proteins prevent the assembly of FtsZ into Z-Ring at that particular space in the cell (34).Once nucleoid gets replicated in to two and separation of nucleoid from each other results in the creation of nucleoid free space. In this nucleoid free space positive regulators overcome the negative regulators so critical concentration of Z-Ring assembly decreases. Once initiation of Z- Ring takes place, more and more FtsZ Proteins are added as it is more likely to add monomers to preassembled protofilaments rather than starting new polymerization. Thus, this cooperatively phenomenon ensures only one Z-Ring formation at medial position. Z-Ring is further strengthened by recruitment of accessory proteins. Interestingly, Z-Ring can be form in a central zone in anucleated Escherichia coli cells, although they are not at precise position, suggesting that the nucleoid is required for normal positioning at mid cell (35).

\section{Model}

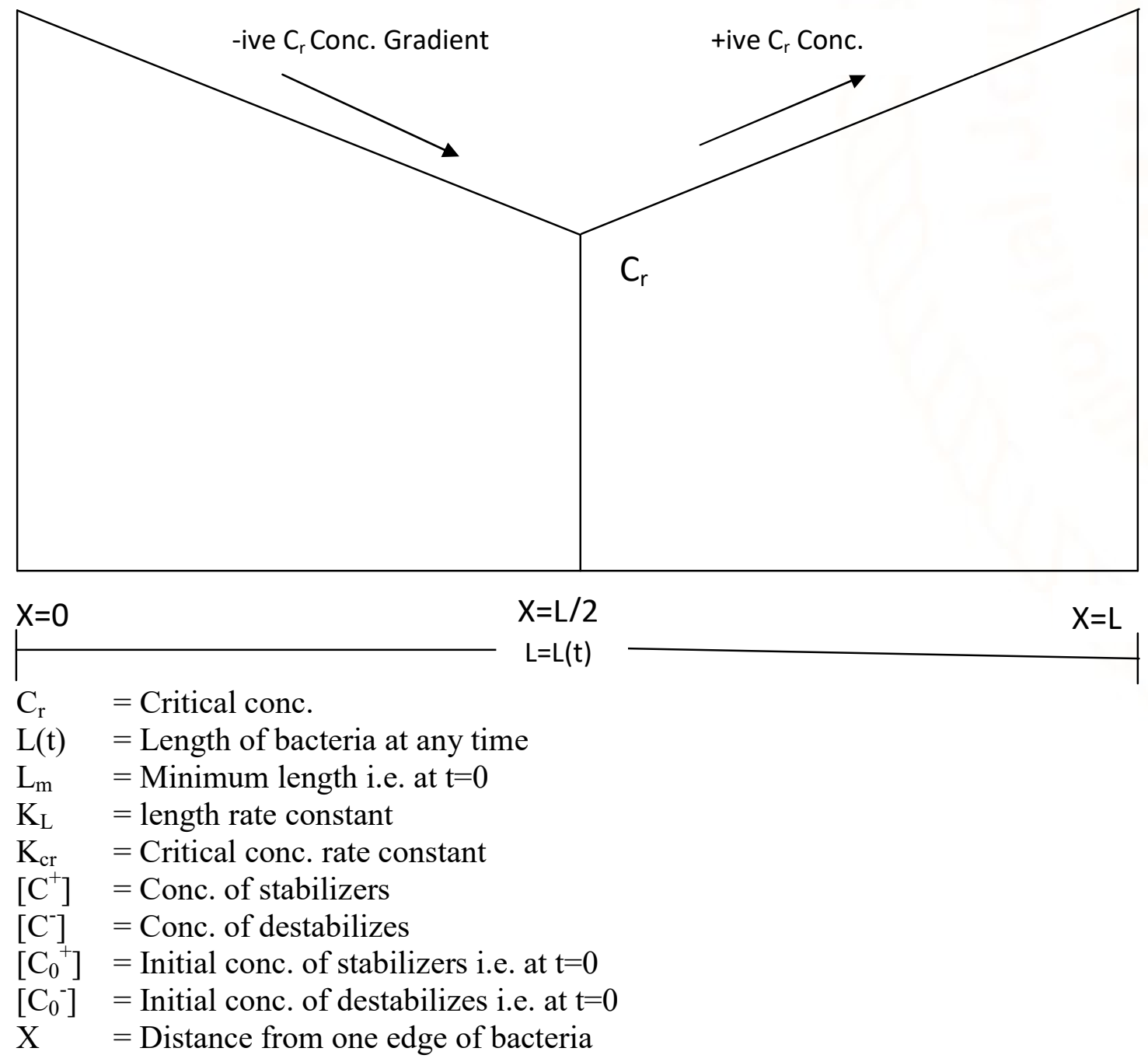




\section{Model equations:}

1. $L(t)=L_{m}+K_{L} * t$

2. for $0 \leq x \leq L / 2$

$$
\begin{gathered}
d \mathrm{C}_{\mathrm{r}} / d \mathrm{x}=-\mathrm{K}_{\mathrm{cr}}\left[\mathrm{C}^{-}\right] /\left[\mathrm{C}^{+}\right] \\
{\left[\mathrm{C}^{+}\right]=\left[\mathrm{C}_{0}^{+}\right] *\left(\mathrm{x}^{\mathrm{t}}+\mathrm{t}\right)} \\
{\left[\mathrm{C}^{-}\right]=\left[\mathrm{C}_{0}^{-}\right] /\left(\mathrm{x}^{\mathrm{t}}+\mathrm{t}\right)}
\end{gathered}
$$

3. for $L / 2<x \leq L$

$$
\begin{gathered}
d \mathrm{C}_{\mathrm{r}} / d \mathrm{x}=\mathrm{K}_{\mathrm{cr}}\left[\mathrm{C}^{-}\right] /\left[\mathrm{C}^{+}\right] \\
{\left[\mathrm{C}^{+}\right]=\left[\mathrm{C}_{0}^{+}\right] /\left(\mathrm{x}^{\mathrm{t}}+\mathrm{t}\right)} \\
{\left[\mathrm{C}^{-}\right]=\left[\mathrm{C}_{0}^{-}\right] *\left(\mathrm{x}^{\mathrm{t}}+\mathrm{t}\right)}
\end{gathered}
$$

\section{DISCUSSION:}

FtsZ is a conserved protein throughout the bacterial kingdom. There is sequence variation of FtsZ from bacteria to bacteria, but C-terminal of FtsZ sequence is almost conserved. The positive regulators of Z-ring assembly are ZipA, ZapA, SepF and FtsA and negative regulators of $Z$-ring assembly are MinCDE, EzrA, Nucleoid occlusions and SulA. Both positive and negative regulators help jointly in regulation of Zring formation. It is know that both positive and negative regulators of Z-ring assembly bind to Cterminal tail of FtsZ. So, there must be tightly regulated competition between facilitators and inhibitors of Z-ring assembly. Increase in FtsZ concentration inside the cell forms extra Z-rings at poles. Thus, FtsZ is a limiting factor in Z-ring formation. So, regulation of cell division depends on concentration of FtsZ. FtsZ concentration can be varied by increasing or decreasing the expression of FtsZ gene, resulting in cell division at appropriate time e.g as in Cauobacter Cresentus there is variation of FtsZ concentration in stalked cells. While in most bacteria the concentration of FtsZ is almost constant throughout the cell cycle. So, regulators of Z-ring assembly are responsible for increasing or decreasing the critical concentration of Z-ring formation, depending on the requirement of cell. Positive regulators help in Z-ring assembly at lesser FtsZ concentration, while negative regulators increase the concentration of FtsZ required for Z-ring assembly. The dynamicity of Z-ring is important for bacterial division. In presence of sodium azide that leads to energy scarcity or at non permissible temperature negative regulators overcome the effect of positive regulators and lead to disassembly of Z-ring formation. The concentration of FtsZ remains almost same during favorable and unfavorable conditions. Once bacteria are subjected to favorable condition reassembly of Z-ring take place.

The distribution of negative regulators like MinCD, EzrA and nucleoid occlusion prevent aberrant cell division at cell poles and during initial stages of nucleoid segregation. Both positive and negative regulators bind to C-terminal of FtsZ. So, we can say there is tug of war between assemblers and dissemblers, which in turn regulate the Z-ring formation. Thus, assembly of Z-ring at a particular cell stage and its position of formation depends on following factors.

a. Concentration of FtsZ

b. Relative affinity of positive and negative regulators to C-terminal of FtsZ, which may depends on charge, hydrophobicity, hydrogen bonding, ionic interactions etc. So there can be regulation through post-translational modification of regulators, may be some of post-translational 
modification increase or decrease the affinity of regulators to FtsZ.

c. Relative abundance of positive and negative regulators: Relative abundance of these proteins depends on their synthesis and degradation. There is variation in positive and negative regulator concentration during bacterial growth, which regulates the Z-ring formation. Localization of EzrA and MinCD at cell poles increase the relative abundance of negative regulators, thus inhibiting cell division at poles.

Understanding of mechanism of bacterial cell division is important in present scenario, as evolution of multidrug resistance bacteria are increasing day by day. Our earlier antibacterial targets were DNA replication, transcription and translation. Early antibiotics have been rendered ineffective by bacteria due to evolution of multidrug resistant pathogens. So we have to search for new targets, bacterial cell division is most appealing drug target. Understanding mechanism of cell division, protein -protein interaction during Z-ring assembly may help us in developing some chemically synthesized analogs and peptides. Those peptides and analogs can be us as antimicrobial agents. In addition they can be used to study the bacterial cell division mechanisms.

\section{REFERENCES}

1) Margolin, W. (2005) FtsZ and the division of prokaryotic cells and organelles, Nat. Rev. Mol. Cell Biol. 6, 862-871.

2) Quardokus, E., Din, N., and Brun, Y. V. (1996) Cell cycle regulation and cell type-specific localization of the FtsZ division initiation protein in Caulobacter, Proc. Natl. Acad. Sci. U. S. A 93, 6314-6319.

3) Adams, D. W. and Errington, J. (2009) Bacterial cell division: assembly, maintenance and disassembly of the $\mathrm{Z}$ ring, Nat. Rev. Microbiol. 7, 642-653.

4) Bork, P., Sander, C., and Valencia, A. (1992) An ATPase domain common to prokaryotic cell cycle proteins, sugar kinases, actin, and hsp70 heat shock proteins, Proc. Natl. Acad. Sci. U. S. A 89, 7290-7294.

5) Lara, B., Rico, A. I., Petruzzelli, S., Santona, A., Dumas, J., Biton, J., Vicente, M., Mingorance, J., and Massidda, O. (2005) Cell division in cocci: localization and properties of the Streptococcus pneumoniae FtsA protein, Mol. Microbiol. 55, 699-711.

6) Feucht, A., Lucet, I., Yudkin, M. D., and Errington, J. (2001) Cytological and biochemical characterization of the FtsA cell division protein of Bacillus subtilis, Mol. Microbiol. 40, 115-125.

7) Shiomi, D. and Margolin, W. (2008) Compensation for the loss of the conserved membrane targeting sequence of FtsA provides new insights into its function, Mol. Microbiol. 67, 558-569.

8) Dai, K. and Lutkenhaus, J. (1992) The proper ratio of FtsZ to FtsA is required for cell division to occur in Escherichia coli, J. Bacteriol. 174, 61456151.

9) Hale, C. A. and de Boer, P. A. (1997) Direct binding of FtsZ to ZipA, an essential component of the septal ring structure that mediates cell division in E. coli, Cell 88, 175-185.

10) Liu, Z., Mukherjee, A., and Lutkenhaus, J. (1999) Recruitment of ZipA to the division site by interaction with FtsZ, Mol. Microbiol. 31, 18531861.

11) Haney, S. A., Glasfeld, E., Hale, C., Keeney, D., He, Z., and de, B. P. (2001) Genetic analysis of the Escherichia coli FtsZ.ZipA interaction in the yeast two-hybrid system. Characterization of FtsZ residues essential for the interactions with ZipA and with FtsA, J. Biol. Chem. 276, 11980-11987.

12) Di, L. G., Fagioli, M., Barionovi, D., Ghelardini, P., and Paolozzi, L. (2003) Use of a two-hybrid assay to study the assembly of a complex multicomponent protein machinery: bacterial septosome differentiation, Microbiology 149, 3353-3359.

13) Errington, J., Daniel, R. A., and Scheffers, D. J. (2003) Cytokinesis in bacteria, Microbiol. Mol. Biol. Rev. 67, 52-65, table.

14) Hale, C. A., Rhee, A. C., and de Boer, P. A. (2000) ZipA-induced bundling of FtsZ polymers mediated by an interaction between $\mathrm{C}$-terminal domains, J. Bacteriol. 182, 5153-5166.

15) Raychaudhuri, D. (1999) ZipA is a MAP-Tau homolog and is essential for structural integrity of the cytokinetic FtsZ ring during bacterial cell division, EMBO J. 18, 2372-2383.

16) Pichoff, S. and Lutkenhaus, J. (2002) Unique and overlapping roles for ZipA and FtsA in septal ring assembly in Escherichia coli, EMBO J. 21, 685693. 
17) Hale, C. A. and de Boer, P. A. (1999) Recruitment of ZipA to the septal ring of Escherichia coli is dependent on FtsZ and independent of FtsA, $J$. Bacteriol. 181, 167-176.

18) Gueiros-Filho, F. J. and Losick, R. (2002) A widely conserved bacterial cell division protein that promotes assembly of the tubulin-like protein FtsZ, Genes Dev. 16, 2544-2556.

19) Ishikawa, S., Kawai, Y., Hiramatsu, K., Kuwano, M., and Ogasawara, N. (2006) A new FtsZinteracting protein, YlmF, complements the activity of FtsA during progression of cell division in Bacillus subtilis, Mol. Microbiol. 60, 13641380.

20) de Boer, P. A., Crossley, R. E., and Rothfield, L. I. (1989) A division inhibitor and a topological specificity factor coded for by the minicell locus determine proper placement of the division septum in E. coli, Cell 56, 641-649.

21) $\mathrm{Hu}, \quad$ Z., Mukherjee, A., Pichoff, S., and Lutkenhaus, J. (1999) The MinC component of the division site selection system in Escherichia coli interacts with FtsZ to prevent polymerization, Proc. Natl. Acad. Sci. U. S. A 96, 14819-14824.

22) de Boer, P. A., Crossley, R. E., and Rothfield, L. I. (1992) Roles of MinC and MinD in the sitespecific septation block mediated by the MinCDE system of Escherichia coli, J. Bacteriol. 174, 6370 .

23) Higashitani, A., Ishii, Y., Kato, Y., and Koriuchi, K. (1997) Functional dissection of a cell-division inhibitor, SulA, of Escherichia coli and its negative regulation by Lon, Mol. Gen. Genet. 254, 351-357.

24) Huang, J., Cao, C., and Lutkenhaus, J. (1996) Interaction between FtsZ and inhibitors of cell division, J. Bacteriol. 178, 5080-5085.

25) Huisman, O. and D'Ari, R. (1981) An inducible DNA replication-cell division coupling mechanism in E. coli, Nature 290, 797-799.

26) Dajkovic, A., Mukherjee, A., and Lutkenhaus, J. (2008) Investigation of regulation of FtsZ assembly by SulA and development of a model for FtsZ polymerization, J. Bacteriol. 190, 25132526.

27) Cordell, S. C., Robinson, E. J., and Lowe, J. (2003) Crystal structure of the SOS cell division inhibitor SulA and in complex with FtsZ, Proc. Natl. Acad. Sci. U. S. A 100, 7889-7894.
28) Mukherjee, A., Cao, C., and Lutkenhaus, J. (1998) Inhibition of FtsZ polymerization by SulA, an inhibitor of septation in Escherichia coli, Proc. Natl. Acad. Sci. U. S. A 95, 2885-2890.

29) Levin, P. A., Kurtser, I. G., and Grossman, A. D. (1999) Identification and characterization of a negative regulator of FtsZ ring formation in Bacillus subtilis, Proc. Natl. Acad. Sci. U. S. A 96, 9642-9647.

30) Haeusser, D. P., Schwartz, R. L., Smith, A. M., Oates, M. E., and Levin, P. A. (2004) EzrA prevents aberrant cell division by modulating assembly of the cytoskeletal protein FtsZ, Mol. Microbiol. 52, 801-814.

31) Levin, P. A., Schwartz, R. L., and Grossman, A. D. (2001) Polymer stability plays an important role in the positional regulation of FtsZ, $J$. Bacteriol. 183, 5449-5452.

32) Haeusser, D. P., Garza, A. C., Buscher, A. Z., and Levin, P. A. (2007) The division inhibitor EzrA contains a seven-residue patch required for maintaining the dynamic nature of the medial FtsZ ring, J. Bacteriol. 189, 9001-9010.

33) Kawai, Y. and Ogasawara, N. (2006) Bacillus subtilis EzrA and FtsL synergistically regulate FtsZ ring dynamics during cell division, Microbiology 152, 1129-1141.

34) Anderson, D. E., Gueiros-Filho, F. J., and Erickson, H. P. (2004) Assembly dynamics of FtsZ rings in Bacillus subtilis and Escherichia coli and effects of FtsZ-regulating proteins, $J$. Bacteriol. 186, 5775-5781.

35) Singh, J. K., Makde, R. D., Kumar, V., and Panda, D. (2007) A membrane protein, EzrA, regulates assembly dynamics of FtsZ by interacting with the C-terminal tail of FtsZ, Biochemistry 46, 1101311022.

36) Levin, P. A. and Losick, R. (1996) Transcription factor Spo0A switches the localization of the cell division protein FtsZ from a medial to a bipolar pattern in Bacillus subtilis, Genes Dev. 10, 478488.

37) Zaritsky, A. and Woldringh, C. L. (2003) Localizing cell division in spherical Escherichia coli by nucleoid occlusion, FEMS Microbiol. Lett. 226, 209-214.

38) Harry, E. J. (2001) Bacterial cell division: regulating Z-ring formation, Mol. Microbiol. 40, 795-803. 